\title{
Chinese culture permeation in the treatment of Parkinson disease: a cross-sectional study in four regions of China
}

\author{
Zhen-Xin Zhang ${ }^{1,2^{*}}$, Honglei Chen ${ }^{3}$, Sheng-Di Chen ${ }^{4}$, Ming Shao ${ }^{5}$, Sheng-Gang Sun ${ }^{6}$, Qiu-Min Qu ${ }^{7}$, \\ Bao-Rong Zhang ${ }^{8}$, Yi-Ming Liu' ${ }^{9}$, Qun Xu ${ }^{10}$, Xia Wan ${ }^{10}$, Ling Li ${ }^{1}$, Hong-Bo Wen ${ }^{1}$, Xia Chen ${ }^{1}$, Hai-Bo Chen ${ }^{11}$, \\ Zhen-Guo Liu ${ }^{12}$, Jian Wang ${ }^{13}$ and Gang Wang ${ }^{4}$
}

\begin{abstract}
Background: Little is known about the clinical features and treatment of Chinese patients with Parkinson disease (PD).

Methods: A large cross-sectional survey of clinical features, medication use, and motor complications was conducted in 901 consecutive PD patients, from 42 randomly selected university-affiliated hospitals in four urban economic regions of China, between December 2006 and May 2007.

Results: The 901 PD patients had age range 30 to 88, and median disease duration 50 months. Most (737, 81.8\%) used L-dopa (median $375 \mathrm{mg} / \mathrm{day}$ ), and often added low doses of other antiparkinsonian agents. Among L-dopa-treated patients, the prevalence of motor complications was low (dyskinesias: $8.5 \%$; motor fluctuations: 18.6\%), even among patients with disease duration $\geq 11$ years (dyskinesias: 18.1\%; motor fluctuations: 42.2\%). Higher L-dopa use was associated with higher occurrence of dyskinesias (OR 2.44; 95\% Cl 1.20-5.13) and motor fluctuations (OR 2.48; 95\% Cl 1.49-4.14). Initiating PD treatment with L-dopa alone (OR 0.46; 95\% Cl 0.22-0.95) or in combination with other medications (OR 0.41; 95\% Cl 0.19-0.87) was associated with less dyskinesia than treatment initiated with non-L-dopa medication.
\end{abstract}

Conclusions: Many Chinese PD patients are treated with low-dose L-dopa and added low-dose antiparkinsonian agents, with a low prevalence of motor complications, which might be influenced by Chinese culture.

Keywords: Parkinson disease, Treatment, Dyskinesias, Motor fluctuations, China

\section{Background}

Parkinson disease (PD) was described in China more than 2400 years ago under the name "wind shaking syndrome," and traditional medications such as the "Jinya wine" and the "tremor pill" were recommended as early as the Tang (581-682 AD) and Ming (1549-1613 AD) dynasties [1]. Chinese culture covers not only material as medications, but also intangible, i.e. traditional ideas, values, and code of conduct. Culture affects generation

\footnotetext{
* Correspondence: wuzhangzhenxin@medmail.com.cn

'Departments of Neurology, Peking Union Medical College Hospital, and Chinese Academy of Medical Sciences, 1 Shuaifuyuan, Wanfujing, Beijing 100730, China

${ }^{2}$ Beijing Brain Health Center, Beijing, China

Full list of author information is available at the end of the article
}

after generation, including doctor and decision maker. Traditional features of Chinese culture permeate the care of the elderly afflicted by chronic neurodegenerative diseases. However, most clinical studies on PD treatment have been conducted in Western countries [2-4]. We therefore conducted a large nationwide survey to characterize the clinical features and treatment of PD patients in China and to compare our results with data from Western countries. Patients were recruited from randomly selected hospitals in seven provincial capital cities representing four economic regions of China with an estimated population of 73.2 million.

\section{Biomed Central}

(c) 2014 Zhang et al.; licensee BioMed Central Ltd. This is an Open Access article distributed under the terms of the Creative Commons Attribution License (http://creativecommons.org/licenses/by/2.0), which permits unrestricted use, distribution, and reproduction in any medium, provided the original work is properly credited. 


\section{Methods}

\section{Design and sampling procedure}

In China, as in the West, most neurology and movement disorder clinics are located in university- affiliated hospitals in large cities. Most patients are either already diagnosed or are referred for diagnostic confirmation. We therefore decided to recruit patients from universityaffiliated hospitals in provincial capitals. This also ensured the quality of PD diagnosis and clinical data collection. We selected seven provincial capitals from four regions in China with various levels of economic and health care development (average $2010 \mathrm{GDP} /$ capita in Chinese Yuan/ medical personnel per 1000 persons in 2009): the Gulf of Bohai (Beijing: 70,234/12.9), Changjiang Delta (Shanghai and Hangzhou: 53,573/9.5 and 5.7), Pearl-River Delta (Guangzhou: 39,978/ 5.0), and Mid-West area (Xian, Wuhan and Jinan: 29,997/4.5, 4.0 and 4.4) [5].

In these regions, there are a total of 349 universityaffiliated teaching hospitals and 42 were randomly selected for the study. The number of participants in each city was estimated a priori, in proportion to its population size. The study team included seven field supervisors, one in each city, and 135 physicians (64 senior neurologists, 46 junior neurologists, and 25 graduate students). PD was diagnosed according to the UK Queen Square Brain Bank diagnostic clinical criteria. Between December 2006 and May 2007, we consecutively recruited 907 individuals with either diagnosed $(n=844)$ or suspected PD $(n=63)$. The principal investigator $(Z Z X)$ reviewed all medical records. Doubtful diagnoses were followed up to one year with additional neurological examinations, and further assessment of the long-term response to L-dopa/DCI, smell test, or brain imaging when necessary. Finally, 901 PD patients were eligible and 6 were excluded due to a final diagnosis of atypical Parkinsonism. All eligible patients agreed to participate and gave written consent. Initial diagnosis and follow-up care was performed at the same participating hospitals in 507 (56.3\%) patients. The other 209 patients were referred from community or district hospitals, with an incorrect initial diagnosis in 66.5\% (139/209). The Ethics Committee on Human Research at the Peking Union Medical College Hospital approved the study.

\section{Data collection}

Clinical data for 901 consecutive PD patients were collected by 142 interviewers in the 42 participating outpatient clinics following a standard protocol. Data collection included basic demographics, family and medical history, past and current treatment, and comorbidities. We also evaluated motor symptoms and signs using standardized instruments such as Hoehn and Yahr Stage (HYS), the Unified Parkinson's Disease Rating Scale I-IV (UPDRS), Mini-Mental State Examination
(MMSE), Hamilton Depression Scale, and pertinent laboratory tests. All interviewers received a two-day training resulting with videotaped interviews in an inter-rater reliability for UPDRS and HYS of more than 0.90 .

\section{Data analyses}

All statistical analyses were performed using SAS (version 8.0) with two-sided alpha of 0.05. In descriptive analyses, we used mean and standard deviation (SD) for normally distributed continuous variables, median and interquartile range (IQ) for continuous variables with skewed distribution, and proportions for categorical variables. We compared demographic and clinical features by disease duration and economic regions. Cardinal variables were compared with the Kruskal-Wallis test, dichotomous variables with $2 \times 4$ Chi-square test. We used multivariate logistic regression to test the relationship between motor complications and demographic and clinical features. Odds ratios (ORs) and 95\% confidence intervals (CIs) were reported accordingly.

\section{Results}

\section{Clinical features and medication uses}

Table 1 shows the demographic and clinical characteristics of 901 Chinese PD patients overall by economic regions. Median age at onset was 61 years. The majority of study participants $(80.8 \%)$ were at HYS-2 or higher, and median disease duration was 50 months for all patients and 70 months for patients with HYS $\geq 2.5$.

At enrollment, L-dopa/DCI was the most commonly used antiparkinsonian drug (737, 81.8\%), followed by dopamine agonists $(296,32.9 \%)$ and amantadine (222, 24.6\%) (Table 2). Among L-dopa/DCI users, 596 (80.9\%) initiated treatment with L-dopa/DCI and at the time of the survey, 498 patients (67.6\%) combined L-dopa/DCI with other antiparkinsonian drugs: dopamine agonists (269, 36.5\%), amantadine $(169,22.9 \%)$, anticholinergics $(126,17.1 \%)$, or selegiline $(75,10.2 \%)$ (Figure 1); including former users of L-dopa/DCI plus these drugs, the percentages increased to $46.1 \%, 41.5 \%, 39.6 \%$, and $17.2 \%$ respectively. Notably, all these drugs were taken at doses lower than those at which they were licensed or recommended for use in published trials from Europe and North America [6]. In our patients, the daily median dose was $375 \mathrm{mg}$ for L-dopa, $200 \mathrm{mg}$ for amantadine, and $5 \mathrm{mg}$ for selegiline, consistently across all regions (Table 2). For dopamine agonists, the median daily doses were $75 \mathrm{mg}$ for piribedil, $0.38 \mathrm{mg}$ for pergolide, $3.75 \mathrm{mg}$ for bromocriptine, and $0.5 \mathrm{mg}$ for pramipexole. At the time of our survey, $32.4 \%$ of the 737 L-dopa/DCI users were receiving single-drug therapy, while $46.1 \%$ were taking two drugs, $17.8 \%$ three drugs, and $3.7 \%$ four or five drugs. The median (IQ) dosage of calculated Ldopa/DCI equivalent daily dose (LED) [7] was 450 (350) 
Table 1 Demographic and clinical characteristics of 901 PD patients

\begin{tabular}{|c|c|c|c|c|c|c|c|c|c|c|c|}
\hline \multirow{2}{*}{$\begin{array}{l}\text { Characteristics } \\
\text { Men, n (\%) }\end{array}$} & \multicolumn{2}{|c|}{$\begin{array}{c}\text { Total } \\
(\mathbf{n}=901)\end{array}$} & \multicolumn{2}{|c|}{$\begin{array}{l}\text { Gulf of Bohai } \\
(n=283)\end{array}$} & \multicolumn{2}{|c|}{$\begin{array}{c}\text { Changjian Delta } \\
(n=275)\end{array}$} & \multicolumn{2}{|c|}{$\begin{array}{l}\text { Pearl-River Delta } \\
(n=110)\end{array}$} & \multicolumn{2}{|c|}{$\begin{array}{c}\text { Mid-west Area } \\
(n=233)\end{array}$} & \multirow{2}{*}{$\begin{array}{r}P \text { Value } \\
0.87\end{array}$} \\
\hline & 562 & $(62.4)$ & 180 & $(63.6)$ & 174 & $(63.3)$ & 67 & $(60.9)$ & 141 & $(60.5)$ & \\
\hline Age at survey (yr) & 67.0 & $(15.0)$ & 68.0 & $(15.0)$ & 66.0 & $(15.0)$ & 67.5 & $(13.0)$ & 67.0 & $(15.0)$ & 0.43 \\
\hline$<60, \mathrm{n}(\%)$ & 254 & $(28.2)$ & 76 & $(26.9)$ & 87 & (31.6) & 27 & $(24.5)$ & 97 & $(41.6)$ & \\
\hline $60-69, n(\%)$ & 266 & $(29.5)$ & 81 & $(28.6)$ & 111 & $(40.4)$ & 36 & $(32.7)$ & 72 & $(30.9)$ & \\
\hline$\geq 70, \mathrm{n}(\%)$ & 381 & $(42.3)$ & 126 & $(44.5)$ & 77 & $(28.0)$ & 47 & $(42.7)$ & 64 & $(27.5)$ & \\
\hline Education (yr) & 10.7 & $(4.6)$ & 12.2 & $(4.2)$ & 10.3 & $(4.5)$ & 9.4 & $(5.1)$ & 10.0 & $(4.3)$ & $<0.001$ \\
\hline Occupation, n (\%) & & & & & & & & & & & $<0.001$ \\
\hline Labor/ Service, n (\%) & 392 & $(43.5)$ & 89 & $(31.5)$ & 146 & $(53.1)$ & 58 & $(52.7)$ & 99 & $(42.5)$ & \\
\hline Administrative, n (\%) & 253 & $(28.1)$ & 107 & $(37.8)$ & 51 & $(18.5)$ & 24 & $(21.8)$ & 71 & $(30.5)$ & \\
\hline Professional, n (\%) & 256 & $(28.4)$ & 87 & $(30.7)$ & 78 & $(28.4)$ & 28 & $(25.5)$ & 63 & $(27.0)$ & \\
\hline Living on personal income, n (\%) & 722 & $(80.1)$ & 261 & $(92.2)$ & 212 & $(77.1)$ & 69 & $(62.7)$ & 180 & $(77.3)$ & $<0.001$ \\
\hline Age of $1^{\text {st }}$ symptom onset (yr) & 61.0 & $(15.0)$ & 60.0 & $(15.0)$ & 60.0 & $(16.0)$ & 61.5 & $(18.0)$ & $63 \cdot 0$ & $(14.0)$ & 0.35 \\
\hline Age at diagnosis (yr) & 62.8 & $(15.6)$ & 61.9 & $(16.0)$ & 61.7 & $(15.6)$ & 63.1 & $(17.8)$ & 63.8 & $(13.5)$ & 0.29 \\
\hline Months from onset to $1^{\text {st }}$ visit & 3.0 & (12.0) & 4.0 & $(12.0)$ & 3.0 & $(9.0)$ & 3.0 & $(12.0)$ & 3.0 & $(12.0)$ & 0.11 \\
\hline Tremor as $1^{\text {st }}$ symptom, $\mathrm{n}(\%)$ & 586 & $(65.4)$ & 185 & $(65.4)$ & 170 & $(61.8)$ & 75 & $(68.2)$ & 156 & $(67.0)$ & 0.55 \\
\hline Resting tremor, n (\%) & 758 & $(84.1)$ & 241 & $(85.2)$ & 233 & $(84.7)$ & 88 & $(80.0)$ & 143 & $(84.1)$ & 0.64 \\
\hline Rigidity, n (\%) & 832 & $(92.3)$ & 270 & $(95.4)$ & 253 & $(92.0)$ & 102 & $(92.7)$ & 207 & $(88.8)$ & 0.05 \\
\hline Bradykinesia, n (\%) & 881 & $(97.8)$ & 281 & $(99.3)$ & 269 & $(97.8)$ & 108 & $(98.2)$ & 223 & $(95.7)$ & 0.05 \\
\hline Hoehn-Yahr stage, n (\%) & & & & & & & & & & & $<0.001$ \\
\hline $1.0-1.5$ & 173 & $(19.2)$ & 44 & $(15.6)$ & 71 & $(18.9)$ & 14 & $(12.7)$ & 44 & $(18.9)$ & \\
\hline $2.0-2.5$ & 560 & $(62.2)$ & 189 & $(66.8)$ & 151 & $(58.4)$ & 84 & $(76.4)$ & 136 & $(58.4)$ & \\
\hline 3.0 & 114 & $(12.6)$ & 34 & $(12.0)$ & 45 & $(12.9)$ & 5 & $(4.6)$ & 30 & $(12.9)$ & \\
\hline $4.0-5.0$ & 54 & $(6.0)$ & 16 & $(5.7)$ & 8 & $(9.9)$ & 7 & $(6.4)$ & 23 & $(9.9)$ & \\
\hline UPDRS-II, scores & 11.0 & $(8.0)$ & 10.0 & $(8.0)$ & 10.0 & $(11.0)$ & 11.0 & $(7.0)$ & 12.0 & $(11.0)$ & $<0.001$ \\
\hline UPDRS-III, scores & 25.0 & $(20.0)$ & 27.0 & $(20.0)$ & 23.0 & $(18.0)$ & 24.0 & $(19.0)$ & 26.0 & $(23.0)$ & 0.02 \\
\hline PD duration, months & 50.0 & $(58.0)$ & 56.0 & $(61.0)$ & 50.0 & $(56.0)$ & 49.0 & $(56.0)$ & 44.0 & $(53.0)$ & $<0.001$ \\
\hline Hoehn-Yahr stage 1.0-2.0 & 37.0 & $(43.0)$ & 41.0 & $(48.0)$ & 40.0 & $(38.0)$ & 37.0 & $(48.0)$ & 27.0 & $(45.0)$ & \\
\hline Hoehn-Yahr stage 2.5-5 & 70.0 & $(70.0)$ & 82.0 & $(78.5)$ & 72.0 & $(69.0)$ & 76.0 & $(75.5)$ & 56.0 & $(53.5)$ & \\
\hline MMSE score < cutoff, n (\%) & 225 & $(25.0)$ & 55 & $(19.4)$ & 61 & $(22.2)$ & 30 & $(27.3)$ & 79 & $(33.9)$ & 0.005 \\
\hline Depressive symptom, n (\%) & 406 & $(45.1)$ & 100 & $(35.3)$ & 113 & (41.1) & 60 & $(54.5)$ & 133 & $(57.1)$ & 0.004 \\
\hline
\end{tabular}

Abbreviations: IQ, interquartile range; Values are given as Median (IQ) unless stated otherwise; MMSE cutoff score for aged 55 and over: $\leq 19$ for illiterate, $\leq 22$ for 1-6 years of education, $\leq 26$ for $\geq 7$ years of education. Depressive symptom: Hamilton Depression Scale (17 items) $\geq 9$. Cardinal variables were compared with the Kruskal-Wallis test, dichotomous variables with $2 \times 4-$ Chi-square test.

$\mathrm{mg} /$ day and differed between regions. LED increased with disease duration. Chinese herbs were used only in 25 mild patients (UPDRS III $=19.1$ ), in 12 as single-drug therapy and in 13 as supplementary therapy.

The four economic regions differed in demographic and PD-related characteristics (Table 1). Compared to patients from other regions, those from Gulf of Bohai were more likely to be professionals or administrators with a higher education level and financial independence. However, they did not differ in age of onset or diagnosis, or main PD symptoms or signs. Mid-West was the least developed region. Compared to this region, PD patients from the Gulf of Bohai had longer disease duration and yet better HYS and activities of daily living (UPDRS-II) scores. This regional difference of disease duration was evident even in stratified analyses by HYS subgroups. The use of amantadine and selegiline was also related to economic development (Table 2).

\section{Disease progression and motor complications}

Of the 737 L-dopa/DCI users, 137 (18.6\%) had motor fluctuations and $63(8.6 \%)$ had dyskinesias. The prevalence of motor complications increased with disease duration (Figure 1): the prevalence of dyskinesia was 3.4\% among patients with 3 years of disease, $6.2 \%$ among patients with 4-6 years of disease as compared to $18.1 \%$ 
Table 2 Medication use of 901 PD patients

\begin{tabular}{|c|c|c|c|c|c|c|c|c|c|c|c|}
\hline \multirow{3}{*}{$\begin{array}{l}\text { Medication use } \\
\mathrm{LED}^{\mathrm{a}}\end{array}$} & \multirow{2}{*}{\multicolumn{2}{|c|}{$\begin{array}{c}\text { Total } \\
(\mathrm{n}=901)\end{array}$}} & \multirow{2}{*}{\multicolumn{2}{|c|}{$\frac{\text { Gulf of Bohai }}{(n=283)}$}} & \multirow{2}{*}{\multicolumn{2}{|c|}{$\begin{array}{c}\text { Changjian Delta } \\
(n=275)\end{array}$}} & \multirow{2}{*}{\multicolumn{2}{|c|}{$\begin{array}{c}\text { Pearl-River Delta } \\
(n=110)\end{array}$}} & \multirow{2}{*}{\multicolumn{2}{|c|}{$\begin{array}{c}\text { Mid-westArea } \\
(n=233)\end{array}$}} & \multirow{3}{*}{$\begin{array}{c}\begin{array}{c}P \\
\text { Value }\end{array} \\
0.01\end{array}$} \\
\hline & & & & & & & & & & & \\
\hline & 450 & (350) & 500 & (330) & 435 & (360) & 525 & (353) & 375 & (330) & \\
\hline LD/DCI treatment, n (\%) & 737 & $(81.8)$ & 227 & $(80.2)$ & 231 & $(84.0)$ & 100 & $(90.9)$ & 179 & $(76.8)$ & 0.01 \\
\hline LD Dose, mg/d & 375 & (265) & 375 & $(200)$ & 375 & $(300)$ & 375 & $(300)$ & 375 & $(250)$ & 0.81 \\
\hline LD/DCI Duration, months ${ }^{b}$ & 29.5 & $(51.0)$ & 36.0 & $(54.0)$ & 34.0 & $(51.0)$ & 21.0 & $(45.0)$ & 16.0 & $(38.0)$ & $<0.001$ \\
\hline Dopamine agonists, n (\%) & 296 & (32.7) & 98 & $(34.6)$ & 83 & $(30.2)$ & 53 & $(48.2)$ & 62 & $(26.6)$ & $<0.001$ \\
\hline Duration, months ${ }^{b}$ & 12.0 & $(28.0)$ & 18.0 & $(32.0)$ & 9.5 & $(23.0)$ & 12.0 & $(21.0)$ & 8.0 & $(19.5)$ & 0.004 \\
\hline Amantadine, n (\%) & 222 & $(24.6)$ & 88 & $(31.0)$ & 77 & $(28.0)$ & 25 & $(22.7)$ & 32 & $(13.7)$ & $<0.001$ \\
\hline Dose, mg/d & 200 & (0) & 200 & $(0)$ & 200 & $(100)$ & 200 & (0) & 200 & $(100)$ & 0.07 \\
\hline Duration, months ${ }^{b}$ & 21.0 & $(43.0)$ & 29.5 & $(62.5)$ & 11.0 & $(34.0)$ & 10.5 & $(21.5)$ & 18.5 & $(53.5)$ & 0.002 \\
\hline Anticholinergics, n (\%) & 169 & $(18.8)$ & 43 & $(15.2)$ & 64 & $(23.3)$ & 15 & $(13.6)$ & 47 & $(20.2)$ & 0.04 \\
\hline Dose, mg/d & 4.0 & (2.0) & 4.0 & $(3.0)$ & 3.0 & $(2.0)$ & 4.0 & $(4.0)$ & 4.0 & $(4.0)$ & 0.02 \\
\hline Duration, months ${ }^{b}$ & 23.0 & $(51.0)$ & 38.0 & $(62.0)$ & 25.0 & $(57.0)$ & 17.0 & $(21.0)$ & 20.2 & $(41.0)$ & 0.23 \\
\hline Entacapone, n (\%) & 55 & (6.1) & 17 & $(6.0)$ & 8 & $(2.9)$ & 11 & $(10.0)$ & 19 & $(8.2)$ & 0.02 \\
\hline Dose, mg/d & 300 & (200) & 300 & $(200)$ & 250 & $(200)$ & 300 & (200) & 300 & $(100)$ & 0.68 \\
\hline Duration, months ${ }^{b}$ & 5.5 & $(14.0)$ & 9.0 & $(14.0)$ & 9.0 & $(20.0)$ & 4.0 & (5.0) & 1.5 & $(6.5)$ & 0.04 \\
\hline Selegiline, n (\%) & 87 & (9.7) & 36 & $(12.7)$ & 36 & $(13.1)$ & 13 & $(11.8)$ & 2 & $(0.9)$ & $<0.001$ \\
\hline Dose, $\mathrm{mg} / \mathrm{d}$ & 5.0 & $(2.5)$ & 5.0 & $(2.5)$ & 5.0 & $(5.0)$ & 5.0 & $(0.0)$ & 5.0 & $(0.0)$ & 0.63 \\
\hline Duration, months ${ }^{b}$ & 15.0 & $(27.0)$ & 24.0 & $(23.0)$ & 12.0 & $(33.0)$ & 7.5 & $(22.0)$ & 15.0 & $(0.0)$ & 0.87 \\
\hline \multicolumn{12}{|l|}{ All LD/DCI users $(n=737)$} \\
\hline Motor fluctuation, n (\%) & 137 & (18.6) & 47 & $(20.7)$ & 50 & $(21.6)$ & 11 & $(11.0)$ & 29 & $(16.2)$ & 0.09 \\
\hline Dyskinesias, n (\%) & 63 & (8.6) & 22 & $(9.7)$ & 15 & $(6.5)$ & 11 & $(11.0)$ & 15 & $(8.4)$ & 0.49 \\
\hline
\end{tabular}

Abbreviations: LED: L-dopa/DCl equivalent daily dose; LD: L-dopa; IQ: Interquartile range.

Values are given as Median (IQ) unless stated otherwise;

${ }^{\mathrm{a}} \mathrm{n}=806$.

${ }^{b}$ Data are available for duration of medication user: $n=726$ for L-dopa, $n=267$ for dopaming agonists, $n=211$ for amantadine, $n=159$ for anticholinergics, $n=46$ for entacapone, and $n=65$ for selegiline.

among patients who had had the disease for $\geq 11$ years; the corresponding numbers for motor fluctuations were $2.9 \%, 14.9 \%$, and $42.2 \%$, respectively. Among young-onset patients $(<50$ years, $\mathrm{n}=111)$, median disease duration was 6.4 years, HYS 2, and the prevalence of dyskinesias and motor fluctuations $12.6 \%$ and $22.5 \%$, respectively. Of the 137 patients with motor fluctuations, 38 had dyskinesias. As a proxy for the severity of motor fluctuations, the median score of UPDRS item 39 (off hours in total waking time) was 1 for both groups of patients.

Although the overall dosages of L-dopa/DCI and total LED were low, they increased with disease duration, from $350 \mathrm{mg} /$ day or $375 \mathrm{mg} /$ day for patients with $<3$ years of the disease to $500 \mathrm{mg} /$ day or $670 \mathrm{mg} /$ day for those with $\geq 11$ years. The dosage of other antiparkinsonian medications remained steadily low throughout the course of the disease, but the proportion of adjuvant medication use increased with disease duration (Figure 1). At enrollment, the proportion of adjuvant therapy in addition to L-dopa/DCI among patients with $<3$ years of the disease was $24.4 \%$ for dopamine agonists, $17.6 \%$ for amantadine, $3.9 \%$ for selegiline, and $10.2 \%$ for anticholinergics. In contrast, the proportions among patients with $\geq 11$ years of disease duration was $49.4 \%$ for dopamine agonists, $28.9 \%$ for amantadine, $16.9 \%$ for selegiline, and $22.9 \%$ for anticholinergics.

In multivariate analyses, HYS $\geq 3$ and L-dopa doses $\geq 400 \mathrm{mg} / \mathrm{d}$ were associated with higher occurrence of dyskinesia (Table 3), whereas initiation of L-dopa/DCI alone or added to other medication was associated with lower occurrence of dyskinesia. PD duration $\geq 5$ years, HYS $\geq 3$, and L-dopa doses $\geq 600 \mathrm{mg} / \mathrm{d}$ were each associated with higher occurrence of motor fluctuations. On the other hand, initiation of treatment with L-dopa/DCI and delayed initiation of treatment one year after onset were not associated with higher occurrence of motor fluctuations.

\section{Discussion}

To the best of our knowledge, this is to date the largest hospital-based clinical survey of PD patients in China. 


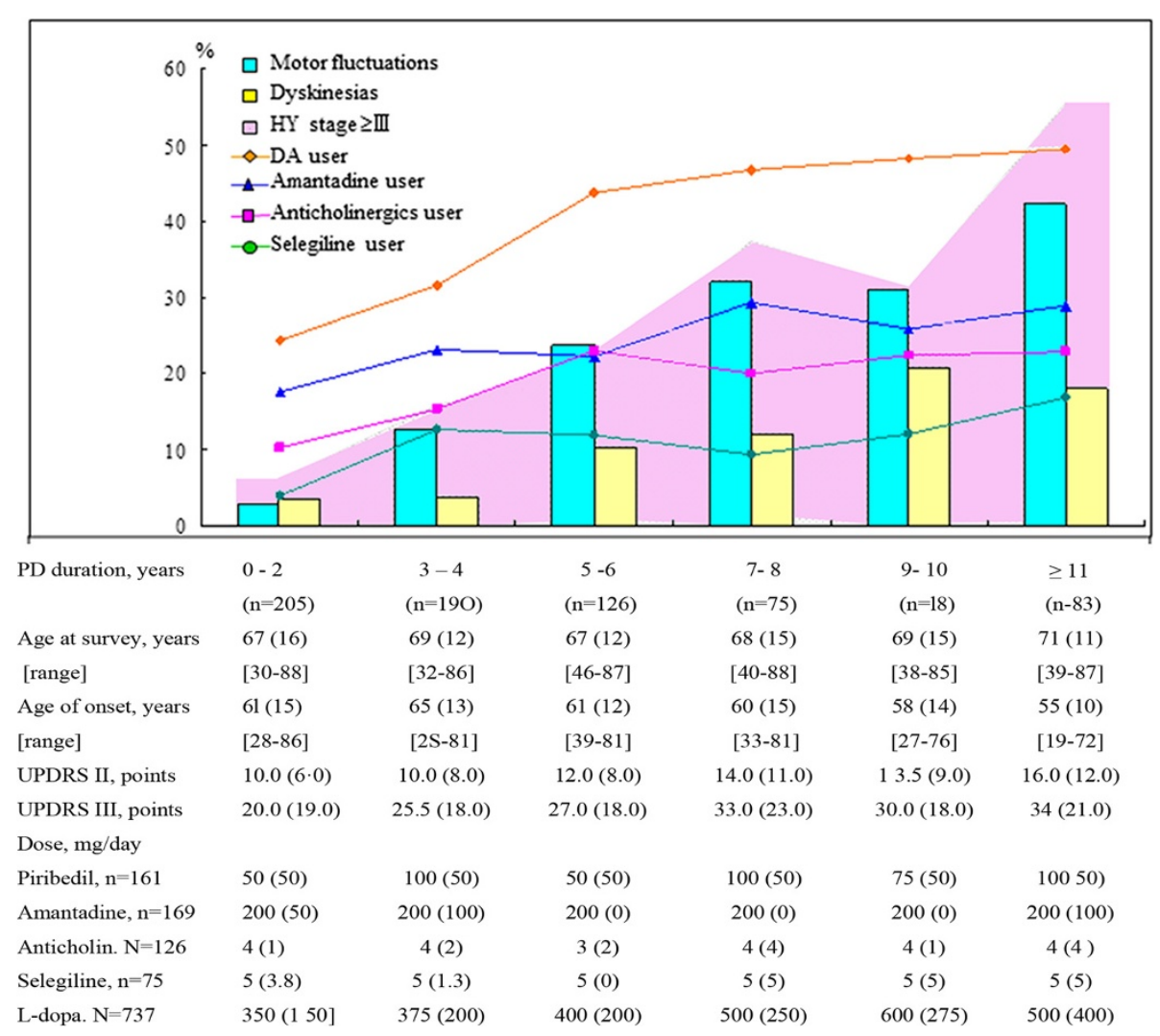

Figure 1 Prevalence of Motor Complications and Medication uses by PD Duration among L-dopa/DCI Users. Abbreviations: Anticholin, Anticholinergics; IQ, Interquartile range; DA, dopamine agonists; UPDRS, Unified Parkinson's Disease Rating Scale; Values are given as median (IQ) unless stated otherwise.

We consider our ambulatory PD population as representative of urban regions in China since sample size was estimated a priori in proportion to regional population size; nearly all patients with neurological symptoms are managed primarily by university outpatient clinics or will be referred to them for final diagnosis and treatment. We may have missed patients with very advanced PD while those with early disease may have been somewhat overrepresented as a consequence of the improvements in the Chinese health assurance and social security system. Comparisons with Western countries therefore need to be made within classes of illness duration. Like their counterparts in Western countries, PD patients in China are predominantly men with average symptom onset in the early sixties; bradykinesia and rigidity are the main signs and most patients are treated with L-dopa/DCI, often in combination with other antiparkinsonian agents [8-13]. Unlike PD patients in Western countries, many Chinese patients take medications at lower dosages and have a lower prevalence of medication-related motor complications.

China is a big country and economic development varies greatly across the country. Our patients were recruited from 42 university-affiliated teaching hospitals randomly selected from all teaching hospitals in seven capital cities in four areas with various levels of economic development. We identified some regional differences in population and clinical characteristics of PD patients that correlated with economic development. Compared to the Mid-West, the least economically developed region, patients in the well-developed Gulf of Bohai were less likely to have HYS $\geq 3$ and had lower UPDRS-II scores, despite a much longer mean disease duration. Patients in the Gulf of Bohai were also more likely to be treated with amantadine and selegiline in addition to L-dopa/DCI.

Studies in Western countries have reported that approximately $50-90 \%$ of PD patients develop motor complications after 5-10 years of dopaminergic replacement therapy [2-4,14-20]. We, however, observed a much lower prevalence of motor complications among Chinese PD patients, overall $8.6 \%$ for dyskinesias and $18.6 \%$ for motor fluctuations. The difference in dyskinesias remained even when comparing patients with similar disease duration (median 4-6 years): 6.2\% in Chinese patients compared to 27.8\% (10.3-40\%) identified by Ahlskog and Muenter in their review article [2] or (median 14 years) $18.1 \%$ of dyskinesias in Chinese patients compared to $58 \%$ in UK patients [20]. The difference in motor fluctuations was 
Table 3 Multivariate regression analyses of motor complications correlates among 737 L-dopa/DCI users

\begin{tabular}{|c|c|c|c|c|c|c|c|}
\hline Variable & & \multicolumn{2}{|c|}{ Dyskinesias } & $P$ Value & \multicolumn{2}{|c|}{ Motor fluctuations } & $P$ Value \\
\hline \multirow[t]{3}{*}{ Age at onset (yr) } & $\geq 59$ & $30 / 420$ & Reference & 0.15 & $68 / 382$ & Reference & 0.13 \\
\hline & $<45$ & $11 / 49$ & $2.32(1.00-5.41)$ & & $14 / 46$ & $1.20(0.57-2.53)$ & \\
\hline & $45-58$ & $22 / 205$ & $1.31(0.70-2.46)$ & & $55 / 172$ & $1.63(1.02-2.61)$ & \\
\hline \multirow[t]{3}{*}{ PD duration (yr) } & $<5$ & 15 / 399 & Reference & 0.36 & $30 / 384$ & Reference & 0.02 \\
\hline & $5-9$ & $28 / 188$ & $1.88(0.79-4.51)$ & & $64 / 152$ & $2.52(1.31-4.85)$ & \\
\hline & $\geq 10$ & $20 / 87$ & $1.82(0.59-5.59)$ & & $43 / 64$ & $2.46(1.05-5.76)$ & \\
\hline \multirow[t]{2}{*}{ Hoehn -Yahr stage } & $<3$ & $38 / 540$ & Reference & 0.03 & $76 / 502$ & reference & $<0.001$ \\
\hline & $\geq 3$ & $35 / 134$ & $2.03(1.08-3.82)$ & & $61 / 98$ & $2.58(1.62-4.11)$ & \\
\hline \multirow[t]{3}{*}{ LD dose, mg/d } & $<400$ & $16 / 370$ & Reference & 0.02 & 39 / 347 & Reference & 0.002 \\
\hline & $400-599$ & $21 / 149$ & $2.48(1.20-5.13)$ & & $37 / 133$ & $1.69(0.99-2.91)$ & \\
\hline & $\geq 600$ & $26 / 155$ & $2.44(1.20-4.98)$ & & $61 / 120$ & $2.48(1.49-4.14)$ & \\
\hline \multirow[t]{3}{*}{ LD/DCI duration (yr) } & $<2$ & 14 / 294 & Reference & 0.44 & $23 / 285$ & Reference & 0.07 \\
\hline & $2-4$ & $15 / 215$ & $1.04(0.47-2.34)$ & & $36 / 194$ & $1.58(0.86-2.87)$ & \\
\hline & $\geq 5$ & $34 / 165$ & $1.73(0.66-4.51)$ & & $78 / 121$ & $2.42(1.14-5.12)$ & \\
\hline Months to initiate & $\leq 12$ & 27 / 339 & Reference & 0.87 & $66 / 300$ & Reference & 0.66 \\
\hline LD/DCl use & $>12$ & $36 / 439$ & $0.95(0.49-1.83)$ & & $71 / 300$ & $0.89(0.54-1.48)$ & \\
\hline \multirow[t]{3}{*}{ Initial treatment } & non LD/DCl & $22 / 119$ & Reference & 0.04 & $35 / 106$ & Reference & 0.42 \\
\hline & LD/DCI only & $22 / 259$ & $0.46(0.22-0.95)$ & & $53 / 228$ & $0.76(0.42-1.36)$ & \\
\hline & $\mathrm{LD} / \mathrm{DCl}+$ others & $19 / 296$ & $0.41(0.19-0.87)$ & & $49 / 266$ & $0.67(0.37-1.22)$ & \\
\hline
\end{tabular}

Abbreviations: LD, L-dopa; $\mathrm{DCl}$, decarboxylase Inhibitor; OR, odds ratios; $\mathrm{Cl}$, confidence interval.

Model adjusted for gender, age at onset, PD duration, Hoehn -Yahr stage, levodoap dose, LD/DCI duration, region, months to initiate LD/DCI use, and initial treatment.

less dramatic, but still $42.2 \%$ compared to $50 \%$ [20]. A similar difference was found when we stratified by age at onset: for example, only $12.6 \%$ of our early-onset cases ( $<50$ years) had dyskinesias compared to $40 \%$ in USA [21]. It is well established that these motor complications are the result of L-dopa treatment $[3,4]$. A notable difference between Chinese patients and those in the West is that Chinese patients took L-dopa (as well as other medications) at low dosages across all regions. Dosages also remained low through the course of the disease. Further, in our study, the presence of dyskinesias was related to the dose of L-dopa, a finding consistent with that of other studies $[3,4]$. Therefore, it is reasonable to suspect that this low prevalence of motor complications may be in part due to low-dose L-dopa/DCI treatment (median $400 \mathrm{mg} / \mathrm{d})$.

In addition to L-dopa/DCI, $67.6 \%$ of Chinese patients also used other antiparkinsonian agents as adjunctive therapy at the time of our survey, also at low doses. As disease duration increased, patients used more classes of these agents, but still at low doses. Dopamine agonists and amantadine were the most commonly used adjunctive medications. Previous studies showed that amantadine might delay the need for L-dopa/DCI in early PD and provide an antidyskinetic effect in advanced patients
[21-24]. In our study, $22.9 \%$ of Chinese PD patients currently used, and $41.5 \%$ had ever used, amantadine combined with L-dopa, a proportion much higher than in Western patients (e.g., current users in the UK 5\%, in Italy 11.4\%, and in Spain 18.8\%) [15,16,25]. Adjunctive selegiline can reduce the need for high-dose L-dopa/DCI and may thus decrease the risk for motor fluctuations, although at higher dosages $(10 \mathrm{mg} / \mathrm{d})$ it may induce dyskinesias $[4,26,27]$. In Europe, the reported proportion of adjunctive selegiline use ranges from 8.1 to $34.4 \%$ $[15,16,27]$. In the present study, $10.2 \%$ of patients currently used, and $17.2 \%$ had ever used, selegiline, and typically at low doses (median $5 \mathrm{mg} / \mathrm{d}$ ). Finally, a recent meta-analysis confirmed that adjuvant therapy with dopamine agonists reduced the need for higher L-dopa/ DCI dose, although it increased dyskinesias in patients with later-stage PD [7]. Dopamine agonists were the most commonly used adjunctive drug among Chinese patients, but again at low doses consistently throughout disease duration. For example, the median dose of piribedil remained at $50-100 \mathrm{mg} /$ day, that of amantadine at $200 \mathrm{mg} /$ day, and that of selegiline at $5 \mathrm{mg} /$ day.

The choice of multiple but low-dose medication may reflect Chinese philosophy regarding the treatment of chronic disease: "a small stream runs far". Chinese 
treatment guideline of PD recommended "never go for full effective dose" [28,29]. Many Chinese patients start Ldopa/DCI in the low therapeutic range. The higher dose that is standard in Western countries may achieve satisfactory motor control sooner, but this may be at the expense of motor complications.

Deferral of L-dopa/DCI treatment has been recommended in order to delay the onset of motor complications in patients younger than 70 years [22]. However, in the current study, motor complications were less frequent in patients who started with L-dopa/DCI alone or in combination with other drugs than in those who started with other drugs. L-dopa/DCI deferral was not associated with a lower occurrence of dyskinesias or motor fluctuations. Similar clinical observations have been made in other studies $[3,4,20,30]$. Therefore, early initiation of low-dose L-dopa/DCI may help to reduce the future occurrence of motor complications. In support of this view, the lifetime cumulative dose of L-dopa use was not related to PD pathology among patients with younger age at onset and longer duration [31]. In summary, early introduction of low-dose symptomatic therapy may preserve basal ganglia compensatory mechanisms $[4,31]$.

A community-based study has recommended using the emergence of axial symptoms (HYS 3) as a surrogate of rapid progression [14]. Compared to studies in the West with similar disease duration, HYS $\geq 3$ disease was not more common in our patients. For example, in patients with a median 14 years disease duration, the average HYS was 3.0 in our study compared with 3.1-3.4 in other studies [20]. in those with about 9 years of disease duration, the average HYS was 2.6 in our population compared to 2.8 in others [11,12]. Median off times in our Chinese patients with motor fluctuations were at a moderate level. Moreover, compared with patients from other regions, those from Bohai had a higher prevalence of antiparkinsonism drug combinations (with a similar low dosage for each form), resulting in higher LED. Patients in Bohai had longer disease duration, especially for more severe subjects (HYS $\geq 2.5$ ), but their activities of daily living were not worse and their quality of life was possibly better, moreover cognitive impairment and depressive symptoms were less frequent than in patients from other areas. Therefore, the low prevalence of motor complications among Chinese patients was not at the expense of poor quality of life, rapid motor deterioration, or prolonged off-times. The Chinese treatment strategy adequately controlled motor signs and helped maintain quality of life.

A multitude of neurotransmitters and receptors, including glutamatergic, opioid, serotonergic, g-aminobutyric acid (GABA)-ergic, adenosine, cannabinoid, adrenergic, histaminergic, and cholinergic systems, have been reported to play a role in the etiology of dyskinesias [32].
The traditional approaches, which usually focus on a single target, may not be appropriate, although nonphysiologic, pulsatile dopamine release seem to play critical roles. Our clinical observation showed that combined antiparkinsonian drugs with different mechanisms of action at a small but effective dose reduced the risk of dyskinesias. It might be explained that the combined treatment targets simultaneously more than one neurotransmitter system, through transcription factors and intracellular signaling to alter expression of dyskinesia [32].

Although this is the largest clinical survey of PD patients in China, our study has several limitations. It is a cross-sectional analysis of patients with various disease durations. Therefore we were unable to make a direct causal inference that low-dose medication leads to fewer motor complications. Although our study is a good reflection of patients from urban areas, we might have missed some patients from rural areas or small cities. However, as explained previously, patients in rural areas of small cities often lack medical resources and have to seek help in large cities for PD diagnosis and treatment. Finally, in China there are no family doctors; familybased home care is the traditional customary, but not institutional care for the elderly. Long-term healthcare is often provided by family members, while patients with long-term PD may see a neurologist less often, with the result that our study may have preferentially included less patients with longer disease duration. However, $42.2 \%$ of our patients were $\geq 70$ years old and $41.0 \%$ had average disease duration of $9.2(5-27)$ years.

\section{Conclusion}

In summary, this large clinical survey provides important data on PD clinical practice in China. The difference observed in medication use and also in the prevalence of motor complications between patients in China and patients in the West may reflect cultural differences in medical treatment. The possibility that low dose L-dopa/DCI use in conjunction with other low-dose antiparkinsonian medications may lead to fewer motor complications should be further investigated in future prospective studies.

\section{Competing interests}

Dr. ZX Zhang, a neurologist at PUMC Hospital, reports no conflicts of interest. Dr. H Chen from the US NIH reports no conflicts of interest. Drs. SD Chen, SG Sun, M Shao, QM Qiu, BR Zhang, and YM Liu, sub-PI from 6 provincial cities, report no disclosures. Drs. X Wan, L Li, X Chen, HB Wen, and Q Xu work at PUMC, and HB Chen in Beijing, and ZG Liu, J Wang and G Wang in Shanghai report no disclosures.

\section{Authors' contributors}

Dr. ZXZ: drafting/revising the manuscript, study concept and design, analysis and interpretation of data, acquisition of data, acquisition of funding, and study supervision. Dr. HC: revising the manuscript, study concept and design, interpretation of data, and acquisition of funding. Dr. XW and QX:

interpretation of data and data statistical analysis. Drs. SDC, MS, SGS, QMQ, $B R Z$, YML, LL, HBW, XC, HBC, ZGL, JW, and GW collected the data. All authors 
contributed to the design of the study, and interpretation of data. All authors read and approved the final manuscript.

\section{Acknowledgements}

This research was supported by 863 -Chinese National Science and Technology Project 2007AA02Z443; and also funded in part by Peking Union Medical College Hospital Project 2006332. Dr. Chen was supported by the intramural research program of the US NIH, the National Institute of Environmental Health Sciences (Z01-ES-101986). We wish to express our sincere gratitude to all study participants for their time and support.

\section{Author details}

${ }^{1}$ Departments of Neurology, Peking Union Medical College Hospital, and Chinese Academy of Medical Sciences, 1 Shuaifuyuan, Wanfujing, Beijing 100730, China. ${ }^{2}$ Beijing Brain Health Center, Beijing, China. ${ }^{3}$ Epidemiology Branch, National Institute of Environmental Health Sciences, National Institutes of Health, Research Triangle Park, North Carolina, USA. ${ }^{4}$ Department of Neurology, Ruijing Hospital, Shanghai Jiaotong University, Shanghai, China. ${ }^{5}$ Department of Neurology, First Affiliated Hospital, Guangzhou Medical College, Guangzhou, China. ${ }^{6}$ Department of Neurology, Xiehe Hospital, Wuhan, China. ${ }^{7}$ Department of Neurology, First Affiliated Hospital of Medical College of Xi'an Jiaotong University, Xi'an, China. ${ }^{8}$ Department of Neurology, Second Affiliated Hospital, Zhejiang University, School of Medicine, Hengzhou, China. ${ }^{9}$ Department of Neurology, First Hospital, Shandong University, Jinan, China. ${ }^{10}$ Department of Epidemiology and Biostatistics, Institute of Basic Medical Science, School of Basic Medicine, Peking Union Medical College, Chinese Academy of Medical Sciences, Beijing, China. ${ }^{11}$ Department of Neurology, Beijing Hospital, Beijing, China. ${ }^{12}$ Department of Neurology, Xinhua Hospital, Shanghai Jiaotong University, Shanghai, China. ${ }^{13}$ Department of Neurology, Huashan Hospital, Shanghai Fudan University, Shanghai, China.

Received: 13 September 2013 Accepted: 31 December 2013 Published: 30 January 2014

\section{References}

1. Zhang ZX, Dong ZH, Román GC: Early descriptions of Parkinson disease in ancient China. Arch Neurol 2006, 63:782-784.

2. Ahlskog JE, Muenter MD: Frequency of levodopa-related dyskinesias and motor fluctuations as estimated from the cumulative literature. Mov Disord 2001, 16:448-458.

3. Olanow CW, Stern MB, Sethi K: The scientific and clinical basis for the treatment of Parkinson disease. Neurology 2009, 72(21 Suppl 4):S1-136.

4. Fahn S, Oakes D, Shoulson I, Kieburtz K, Rudolph A, Lang A, Olanow CW, Tanner C, Marek K, Parkinson study group. : Levodopa and the progression of Parkinson's disease. N Engl J Med 2004, 351:2498-2508.

5. Zhang S (Ed): Chinese Statistical Yearbook. Beijing: China Statistical Publishing House; 2010.

6. Stowe R, Ives N, Clarke CE, Handley K, Furmston A, Deane K, van Hilten JJ, Wheatley K, Gray R: Meta-analysis of the comparative efficacy and safety of adjuvant treatment to levodopa in later Parkinson's disease. Mov Disord 2011, 26:587-598.

7. Tomlinson CL, Stowe R, Patel S, Rick C, Gray R, Clarke CE: Systematic review of levodopa dose equivalency reporting in Parkinson's disease. Mov Disord 2010, 25:2649-2653.

8. Kang GA, Bronstein JM, Masterman DL, Redelings M, Crum JA, Ritz B: Clinical characteristics in early Parkinson's disease in a central California population-based study. Mov Disord 2005, 20:1133-1142.

9. Lees AJ, Hardy J, Revesz T: Parkinson's disease. Lancet 2009, 373 (9680):2055-2066.

10. Marttila RJ, Rinne UK: Epidemiology of Parkinson's disease in Finland. Acta Neurol Scand 1976, 53:81-102.

11. Alves $G$, Wentzel-Larsen T, Aarsland D, Larsen JP: Progression of motor impairment and disability in Parkinson disease a population-based study. Neurology 2005, 65:1436-1441.

12. Schrag A, Dodel R, Spottke A, Bornschein B, Siebert U, Quinn NP: Rate of clinical progression in Parkinson's disease: A prospective study. Mov Disord 2007, 22:938-945.

13. Riedel O, Klotsche J, Spottke A, Deuschl G, Förstl H, Henn F, Heuser I, Oertel W, Reichmann H, Riederer P, Trenkwalder C, Dodel R, Wittchen HU: Cognitive impairment in 873 patients with idiopathic Parkinson's disease. Results from the German Study on Epidemiology of Parkinson's Disease with Dementia (GEPAD). J Neurol 2008, 255:255-264.

14. Evans JR, Mason SL, Williams-Gray CH, Foltynie T, Brayne C, Robbins TW, Barker RA: The natural history of treated Parkinson's disease in an incident, community based cohort. J Neurol Neurosurg Psychiatry 2011, 82:1112-1118.

15. Schrag A, Quinn N: Dyskinesias and motor fluctuations in Parkinson's disease. A community- based study. Brain 2000, 123:2297-2305.

16. López IC, Ruiz PJG, del Pozo SVF, Bernardos VS: Motor complications in Parkinson's disease: Ten year follow-up study. Mov Disord 2010, $25: 2735-2739$

17. Hely MA, Morris JGL, Reid WGJ, Trafficante R: Sydney multicenter study of Parkinson's disease: Non-L-dopa-responsive problems dominate at 15 years. Mov Disord 2005, 20:190-199.

18. Rascol O, Brooks DJ, Korczyn AD, De Deyn PP, Clarke CE, Lang AE: A five year study of the incidence of dyskinesia in patients with early Parkinson's disease who were treated with ropinirole or levodopa. N Engl J Med 2000, 342:1484-1491.

19. Benbir G, Özekmekçi S, Apaydin H, Delil S, Erginöz E: A hospital-based study: Risk factors in development of motor complications in 555 Parkinson's patients on levodopa therapy. Clin Neurol Neurosurg 2006, 108:726-732.

20. Katzenschlager R, Head J, Schrag A, Ben-Shlomo Y, Evans A, Lees AJ, on behalf of the Parkinson's Disease Research Group of the United Kingdom: Fourteen-year final report of the randomized PDRG-UK trial comparing three initial treatments in PD. Neurology 2008, 71:474-480.

21. Kumar N, Van Gerpen JA, Bower JH, Ahlskog JE: Levodopa-dyskinesia incidence by age of Parkinson's disease onset. Mov Disord 2005, 20:342-366.

22. Olanow CW, Watts RL, Koller WC: An algorithm (decision tree) for the management of Parkinson's disease (2001): Treatment Guidelines. Neurology 2001, 56(11 suppl 5):S1-S88.

23. Ahmed I, Bose SK, Pavese N, Ramlackhansingh A, Turkheimer F, Hotton G, Hammers A, Brooks DJ: Glutamate NMDA receptor dysregulation in Parkinson's disease with dyskinesias. Brain 2011, 134:979-986.

24. Wolf E, Seppi K, Katzenschlager R, Hochschorner G, Ransmayr G, Schwingenschuh P, Ott E, Kloiber I, Haubenberger D, Auff E, Poewe W: Long-term antidyskinetic efficacy of amantadine in Parkinson's disease. Mov Disord 2010, 25:1357-1363.

25. Barone P, Antonini A, Colosimo C, Marconi R, Morgante L, Avarello TP, Bottacchi E, Cannas A, Ceravolo G, Ceravolo R, Cicarelli G, Gaglio RM, Giglia RM, lemolo F, Manfredi M, Meco G, Nicoletti A, Pederzoli M, Petrone A, Pisani A, Pontieri FE, Quatrale R, Ramat S, Scala R, Volpe G, Zappulla S, Bentivoglio AR, Stocchi F, Trianni G, Dotto PD: The Priamo Study: A multicenter assessment of nonmotor symptoms and their impact on quality of life in Parkinson's disease. Mov Disord 2009, 24:1641-1649.

26. Zhao YJ, Wee HL, Au WL, Seah SH, Luo N, Li SC, Tan LCS: Selegiline use is associated with a slower progression in early Parkinson's disease as evaluated by Hoehn and Yahr Stage transition times. Parkinsonism Relat Disord 2011, 17:194-197.

27. Pålhagen $\mathrm{S}$, Heinonen E, Hägglund J, Kaugesaar T, ki-lkola O, Palm R, the Swedish Parkinson Study Group: Selegiline slows the progression of the symptoms of Parkinson disease. Neurology 2006, 66:1200-1206.

28. Neurology CMA: Proposal in Treatment of Parkinson disease. Chin I Neurol 1999, 32:237-238.

29. Chin Med Asso Neurology: Movement disorder and Parkinson disease. Treatment guideline of Parkinson disease. Chin J Neurol 2006, 39:409-412.

30. Cedarbaum JM, Gandy SE, McDowell FH: "Early" initiation of levodopa treatment does not promote the development of motor response fluctuations, dyskinesias, or dementia in Parkinson's disease. Neurology 1991, 41:622-629.

31. Parkkinen L, O'Sullivan SS, Kuoppamäki M, Collins C, Kallis C, Holton JL, Williams DR, Revesz T, Lee AJ: Does levodopa accelerate the pathologic process in Parkinson disease brain? Neurology 2011, 77:1420-1426.

32. Huot $P$, Johnston $T H$, Koprich JB, Fox SH, Brotchie JM: The pharmacology of L-DOPA-induced dyskinesia in Parkinson's disease. Pharmacol Rev 2013, 65:171-222

doi:10.1186/1756-0500-7-65

Cite this article as: Zhang et al:: Chinese culture permeation in the treatment of Parkinson disease: a cross-sectional study in four regions of China. BMC Research Notes 2014 7:65. 